\title{
SEMI-ANALYTICAL AND SEMI-NUMERICAL METHODS IN CELESTIAL MECHANICS
}

\author{
SLAWOMIR BREITER \\ Astronomical Observatory, Adam Mickiewicz University \\ Poznań, Poland
}

\begin{abstract}
Many quite different theories and methods of celestial mechanics are referred to as semi-analytical or semi-numerical. Their common feature is the trade-off of a generality for a computational efficiency. Four main ideas behind semi-analytical theories are identified and reviewed: series contraction, analytical support of numerical integration, piecewise solutions, and numerical evaluation of action-angle variables. The role of numerical analysis tools in analytical theories is briefly discussed.
\end{abstract}

\section{Introduction}

The great and frequently quoted discovery of Molière's Mr. Jourdain, that he was speaking prose all through his life, originated from a sharp dichotomy imposed by his tutor: prose or poetry and no other way. One does not have to be an expert in literature to judge such a distinction as instructive but rather oversimplified. We can well imagine the same kind of tutor explaining to a modern Mr. Jourdain that theories of motion in celestial mechanics are either numerical or analytical. In this statement the two extremes are clearly identified: the analytical "poetry" explains the nature of trajectories and their dependence upon physical parameters and initial conditions, still struggling with the limitations imposed by the language of perturbation series; the numerical "prose" generates single trajectories step by step in a "sledgehammer approach" (Fox, 1984) - quite often more efficiently than the analytical methods do, but the more information we want about what is behind the generated sequence of numbers, the more numerical analysis tools must be used. To approach the explanation level of analytical theories one has to integrate numerically not only the equations of motion for a large family of initial conditions, but also their variational equations - and all this must be followed by a fair amount of numerical harmonic analysis.

\section{M. Wytrzyszczak, J. H. Lieske and R. A. Feldman (eds.),}

Dynamics and Astrometry of Natural and Artificial Celestial Bodies, 411, 1997.

(C) 1997 Kluwer Academic Publishers. Printed in the Netherlands. 
But, in between the two extremes there lies a huge realm of the theories and methods dubbed semi-analytical or semi-numerical. This "poetic prose" and these "epic poems" of celestial mechanics have always been the domain of ingenious stratagems in quest of an efficient compromise between functions and numbers. The main goal of this presentation is to collect the achievements in the form of a tool-box - mainly to guide the newcomers, but also to help looking for yet unexplored possibilities.

\section{What Does it Mean Semi-Analytical ?}

The disorder in terminology concerning semi-analytical methods is almost frightening. Not only the expressions "semi-analytical" and "semi-numerical" are used either as synonyms or as distinct words, but also some authors try to avoid them writing about "partly numerical", "almost numerical", "semi-general" or "step by step" methods. Some obviously semi-analytical theories are named not only "analytical" but even "totally analytical". The attempt of Chapront-Touzé (1982) to regulate the terminology has not remedied the chaos. Hence, for the purpose of the present review, only the word "semi-analytical" will be used [save for the method of Henrard (1990)] to avoid additional confusion.

Let us call semi-analytical the methods which are essentially based on general perturbation theory, but which involve some techniques eliminating partially the information about the dependence of motion upon initial conditions and physical parameters, or additionally restricting the domain of validity of the obtained solution. This loss of generality - usually caused by the use of some numerical analysis tool - leads to some gain in the efficiency for particular trajectories to be described. To see it better, let us look first at the four main branches of semi-analytical theories, grouped and named here according to their leading ideas: series contraction, analytical support of numerical integration, piecewise solutions, and numerically evaluated action-angle variables. After these four, already classical themes, some more numerical assistance tools will be given in short.

\section{Series Contraction}

A generic form of series arising in perturbation theory may be expressed as

$$
\begin{aligned}
& \sum_{m, n, p} N_{m, n, p} \prod_{i} \varepsilon_{i}^{m_{i}} \prod_{j} \alpha_{j}^{n_{j}} \begin{array}{l}
\text { sin } \\
\text { cos }
\end{array}\left[\sum_{k} p_{k} \beta_{k}\right], \\
& \beta_{k}=\omega_{k}(\varepsilon, \alpha) t+\beta_{k, 0}, \quad \alpha_{i}=\text { const. }
\end{aligned}
$$

$N_{m, n, p}$ is a numerical coefficient, $\varepsilon$ are physical parameters (some of them being small parameters in a mathematical sense), $\alpha$ and $\beta$ are metrical and angular variables respectively, and frequencies $\omega$ are also power series of $\varepsilon$ and $\alpha$. As referred to the above series, the word "long" is usually a subtle 
euphemism. To get them shorter - and so easier to derive and evaluate one can do the following:

1. Substitute numerical values for some of the parameters or metrical variables; this removes the relevant indices from sums and shortens the products. The approach is classical and has a long tradition [see Chapront-Touzé (1982)]. At the extreme, we may come to the series

$$
\begin{aligned}
& \left.\sum_{p} N_{p} \begin{array}{l}
\sin \\
\cos
\end{array} \sum_{k} p_{k} \beta_{k}\right], \\
& \beta_{k}=N_{k}^{\prime} t+\beta_{k, 0},
\end{aligned}
$$

valid only for a particular orbit perturbed by particular forces. This type of compression can be done in a more subtle way, by substituting $\alpha=\alpha_{N}(1+\delta \alpha)$ or $\varepsilon=\varepsilon_{N}(1+\delta \varepsilon)$, where numerical values are given to $\alpha_{N}$ and $\varepsilon_{N}$. For $\delta \alpha$ and $\delta \varepsilon$ small enough, we may obtain series which are much shorter from the original (1) and (2), but they still hold their analytical character for a restricted class of orbits. This is the simplest way of overcoming the slow convergence due to high eccentricities in asteroid theory (Ferraz-Mello, 1987) or high mean motions ratio in lunar theory (Henrard, 1978).

2. Decompose trigonometric terms into products of rapidly oscillating and slowly oscillating parts. The sine/cosine of the slowly changing angles can be approximated by Taylor series or Chebyshev polynomials of time. The former approach leads to classical Poisson terms of a planetary theory; the latter was proposed by Chapront $(1982,1984)$. Many long-periodic contributions can merge into a small number of terms if their frequencies are given numerically. In this case the loss concerns the "orbital information" and also the length of the time span on which the theory is valid.

Of course, these two methods can be applied together, and in their most radical version - when the entire trigonometric terms are approximated by time polynomials - the result looks exactly like a synthetic theory obtained from numerical integration.

\section{Analytical Support of Numerical Integration}

The class of theories considered in this section, merging numerical integration with analytical series, demonstrates how fruitful can be the union of the seemingly opposite techniques. Numerical integration of motion can be accelerated even some tens or hundreds of times if good use is made of general perturbation methods. Let us enumerate the main possibilites of achieving the speed up and reducing the accumulation of errors of numerical integration: 
1. "analytical complements": The numerical integration of the Solar System consists of the N-body problem (the Sun and interacting planets), and of minor contributions due to the asteroids, the Moon, general relativity etc. Bretagnon (1986) gained much of computation time integrating numerically only the former problem, and adding the perturbations due to the latter derived from analytical solutions. Note, that these perturbations are small enough to be expressed by short first-order series.

2. analytical filtering: The main idea behind this type of methods comes from the distinction between long-periodic and short-periodic variations worked out in analytical theories. Transforming the equations of motion by averaging, one obtains a slowly evolving system; the system which would cause some problems in further analytical treatment, but which can be integrated numerically quite fast due to a large stepsize allowed. According to the way of averaging three cases arise:

(a) The equations can be averaged by analytical integration; this way, one can go far in powers of a small parameter, reaching even high accuracy. The best examples are the planetary theory of Laskar (1990) and the artificial satellite theories of Cefola et al. [see Danielson (1994)] and of Métris and Exertier (1995). Short periodic perturbations resulting from the transformation may be computed from analytical expressions if they are of any interest in the studied problem.

(b) The equations can be averaged by numerical quadratures between subsequent steps of numerical integration. The steps can be long - covering one or more orbital periods. This approach is known as "Schubart averaging" [see Schubart (1968) and Moons (1994) for more details].

(c) The averaging by numerical quadratures can be performed before starting the numerical integration, but in this case one should store a large grid of the numerical values of the right-hand sides, as functions of slow variables. An example of this approach is the tesseral resonance study by Nacozy and Diehl (1982).

The great advantage of averaging through numerical quadratures is the possibility of treating large eccentricities and inclinations. However, in the applications known to the author, this averaging gives the first-order transformation with no way to evaluate the short-periodic terms. The question of passing to higher orders (and of doing it efficiently) remains open.

One thing should be emphasized here, because it is frequently overlooked in applications of averaged equations: averaged equations generate a mean trajectory which actually may have some offset with respect to the real trajectory smoothed out numerically (or by any other means). For the way 
of removing this offset without computing short periodic terms (where it is usually hidden) - see Métris and Exertier (1995).

\section{Piecewise Analytical Treatment}

Under more or less severe restrictions, one can solve the equations of motion on the interval ranging from a fraction of orbital period to a couple of revolutions. After a single step the variables are updated with the analytically derived corrections and the procedure is repeated. This strategem is particularly useful when one of the perturbing forces is discretely modeled. One can recognize here the principle of numerical integration, but general perturbation theory and expansions in powers of small parameter are still involved in this semi-analytical approach.

The method is rather widespread, especially in artificial satellite problems with drag or shadow. In the former, complicated expressions for longterm drag contribution are avoided [eg. Watson et al. (1975)]; in the latter, the discontinuity of the shadow function is by-passed thanks to splitting the solution into sunlit and shaded parts [eg. Lautman (1977)]. The "stroboscopic method" of Roth (1979), which determines the elements at subsequent peri- or apocenter passages, is also based on the principles discussed here.

Although it may be controversial, the explicit symplectic integrator of Wisdom and Holman (1991) - as interpreted by Saha and Tremaine (1992) - can also be understood as a semi-analytical method. The Hamiltonian is separated into the Keplerian part $\mathcal{H}_{0}$ and a perturbation $\varepsilon \mathcal{H}_{1}$. One step of the integrator is a composition of two exact, analytical solutions of the equations resulting from $\mathcal{H}_{0}$ and $\varepsilon \mathcal{H}_{1}$ alone, and its error is proportional to the product of $\varepsilon$ and some power of the stepsize.

\section{Numerical Transformation to Action-Angle Variables}

There are many problems in celestial mechanics where the system of averaged equations still has at least two degrees of freedom and a nontrivial structure with libration zones and separatrices; the best examples are resonance problems. If the Hamiltonian is a sum of the separable part $\mathcal{H}_{0}$ and a perturbation $\varepsilon \mathcal{H}_{1}$

$$
\mathcal{H}=\mathcal{H}_{0}\left(q_{1}, q_{2}, p_{1}, p_{2}\right)+\varepsilon \mathcal{H}_{1}\left(q_{1}, q_{2}, p_{1}, p_{2}\right)
$$

a nice way of studying the phase flow would be the transformation from $q, p$ to the action-angle variables $J, \psi$ admitted by $\mathcal{H}_{0}$, with the Hamiltonian $\mathcal{H}$ turned into

$$
\mathcal{K}=\mathcal{K}_{0}\left(J_{1}, J_{2}\right)+\varepsilon \mathcal{K}_{1}\left(J_{1}, J_{2}, \psi_{1}, \psi_{2}\right) .
$$

The perturbing part $\mathcal{K}_{1}$ takes the form of Fourier series in $\psi$ with amplitudes depending on $J$. This transformation, however, usually involves a fair 
amount of algebra and may be inexpressible in terms of elementary functions. Henrard (1990) overpowered the difficulty by resorting to numerical methods. In his approach - which has almost gained the position of the semi-analytical (or rather the semi-numerical) method - the values of actions $J(q, p)$ are obtained by numerical integration of the equations admitted by $\mathcal{H}_{0}(q, p)$, along a closed trajectory. The expression of $\mathcal{K}_{1}$ is then supplied by numerical harmonic analysis. The method has been applied and further developed in resonant problems of asteroids (Morbidelli and Henrard, 1991) and of artificial satellites (Delhaise and Henrard, 1993), where high inclinations and/or eccentricities are involved. The numerical transformation to action-angle variables opened the way to an ingenious revitalisation of Delaunay's perturbation theory by Morbidelli (1993), which permits to obtain better approximation than the first-order method of Henrard.

\section{Numerical Assistance Tools in Analytical Theories}

In the preceding sections, we have presented four - already classical lines along which semi-analytical theories are built. But the concept of mixing analytical and numerical methods is so prolific, that we are still far from completing the review. Let us see now, how some methods of numerical analysis help to break through intricasies of analytical theories. We have already met numerical integration, numerical quadratures, harmonic analysis and polynomial approximation grafted on general perturbation constructions. Glancing through the multitude of theories we meet some of them again in different places:

1. Numerical quadratures: quite useful in "piecewise analytical" theories when analytical quadratures become complicated (Hoots and France, 1987), they may also serve to evaluate the values of special functions like Hansen's functions or Laplace coefficients (Gooding and King-Hele, 1988).

2. Fourier transforms and frequency analysis: powerful techniques helping to develop a disturbing function (Brown and Shook, 1933) and to retrieve qualitative information from numerically integrated trajectory (Laskar, 1993).

3. Polynomial approximation and interpolation: indirectly involved in most of numerical methods, but in some applications they help much in situations where due to the use of numerical quadratures or other means one gets a function defined by the set of its values.

4. Iterative root finding: Newton-Raphson or direct iterations are frequently used in the disturbing function's development where one starts with approximate series (Kovalevsky, 1963); but their more "semianalytical" use is to get rid of the problems caused by coupling of different perturbations. The example can be found in the "piecewise 
analytical" theory of artificial satellite with atmospheric drag of Watson et al. (1975). The contribution due to the drag depends on the trajectory affected by the oblateness perturbations and vice versa. The problem is easily solved by iterating the two seprate perturbation expressions and updating variables after each step until the required accuracy is reached.

5. Numerical differentiation: applied mainly to compensate the information loss in compressed series or numerical quadratures (Kubo, 1982; Hoots and France, 1987); this tool, however, seems to be yet unappreciated in less straightforward applications.

6. Linear algebraic systems solving: If the solution method is the substitution of series with undetermined coefficients into the equations of motion, the resulting system of linear equations can be solved by standard numerical routines like in Diez et al. (1991).

7. Runge-Kutta approximation: Last, but not least, come unusual ways of using the so called "Runge-Kutta expansions" (Kizner, 1964). Kizner was the first to observe, that power series of a small parameter $\varepsilon$ arising in Poisson's perturbation method can be replaced by a step of a Runge-Kutta method solving a variational problem with $\varepsilon$ as the independent variable, and followed by a usual integration with respect to time. This apparently forgotten idea was rediscovered by the author (Breiter, 1996) in the context of Lie transformations, where it occurs even more naturally and may be generalised for other numerical integration methods.

\section{Conclusions}

The development of the large variety of semi-analytical methods was possible thanks to the advances in computer technology. Fortunately, the alleged contradiction between numerical and analytical methods has not resulted in their clash, but it lets both sides draw from the treasures' chest of the supposed opponent. Although one would complain, that the term "semianalytical" has become too ambiguous - due to the multitude of different methods it covers - let us hope, that this ambiguity will grow each year, resulting in more and more ingenious theories of motion.

\section{References}

Breiter, S.: 1996, "On the numerical transformation of variables in perturbation theory", Celest. Mech. \& Dyn. Astron., in press.

Bretagnon, P.: 1986, "Construction of a planetary solution with the help of an N-body program, and analytical complements", Celest. Mech. 38, 181-190.

Brown, E.W. and Shook, C.A.: 1933, Planetary Theory, Cambridge University Press, London.

Chapront, J.: 1982, "The Fourier-Chebyshev approximation for time series with a great many terms", Celest. Mech. 28, 415-430. 
Chapront, J.: 1984, "Approximation methods in celestial mechanics", Celest. Mech. 34, $165-184$.

Chapront-Touze, M.: 1982, "Progress in the analytical theories for the orbital motion of the Moon", Celest. Mech. 26, 53-62.

Danielson, D.A.: 1994, "Semianalytic satellite theory: second-order expansions in the true longitude $L^{\prime \prime}$, in: Advances in the Astronautical Sciences (Misra, A.K. et al., eds), 85, $2457-2474$.

Delhaise, F. and Henrard, J.: 1993, "The problem of critical inclination combined with a resonance in mean motion in artificial satellite theory", Celest. Mech. \& Dyn. Astron. 55, 261-280.

Diez, C., Jorba, A., and Simó, C.: 1991, "A dynamical equivalent to the equilateral libration points of the Earth-Moon system", Celest. Mech. E Dyn. Astron. 50, 13.

Ferraz-Mello, S.: 1987, "Expansion of the disturbing force-function for the study of higheccentricity librations", Astron. Astrophys. 183, 397-402.

Fox, K.: 1984, "Numerical integration of the equations of motion of celestial mechanics", Celest. Mech. 33, 127-142.

Gooding, R.H. and King-Hele, D.G.: 1988, "Explicit forms of some functions arising in the analysis of resonant satellite orbits", R.A.E. Tech. Rep., No 99035.

Henrard, J.: 1978, "Hill's problem in lunar theory", Celest. Mech. 17, 195-204.

Henrard, J.: 1990, "A semi-numerical perturbation method for separable Hamiltonian systems", Celest. Mech. \& Dyn. Astron. 49, 43-68.

Hoots, F.R. and France, R.G.: 1987, "An analytic satellite theory using gravity and a dynamic atmosphere", Celest. Mech. 40, 1-18.

Kizner, W.: 1964, "A high-order perturbation theory using rectangular coordinates", Celestial Mechanics and Astrodynamics (V.G. Szebehely, ed.), Academic Press, New York, 81-99.

Kovalevsky, J.: 1963, Introduction à la Mécanique Céleste, Armand Colin, Paris.

Kubo, Y.: 1982, "Perturbations by the oblatness of the Earth and by the planets in the motion of the Moon", Celest. Mech. 26, 97-112.

Laskar, J.: 1990, "The chaotic motion of the Solar system: A numerical estimate of the size of chaotic zones", Icarus 88, 266-291.

Laskar, J.: 1993, "Frequency analysis of a dynamical system", Celest. Mech \& Dyn. Astron. 56, 191-196.

Lautman, D.A.: 1977, "Perturbations of a close-Earth satellite due to sunlight diffusely reflected from the Earth. I: Uniform albedo", Celest. Mech. 15, 387-420.

Métris, G. and Exertier, P.: 1995, "Semi-analytical theory of the mean orbital motion", Astron. Astrophys. 294, 278-286.

Moons, M.: 1994, "Extended Schubart averaging", Celest. Mech. \& Dyn. Astron. 60, 173.

Morbidelli, A.: 1993, "On the succesive elimination of perturbation harmonics", Celest. Mech. \& Dyn. Astron. 55, 101-130.

Morbidelli, A. and Henrard, J.: 1991, "Secular resonances in the asteroid belt: Theoretical perturbation approach and the problem of their location", Celest. Mech. \& Dyn. Astron. 51, 131-168.

Nacozy, P.E. and Diehl, R.E.: 1982, "Long-term motion of resonant satellites with arbitrary eccentricity and inclination", Celest. Mech. 27, 375-398.

Roth, E.A.: 1979, "On the higher-order stroboscopic method", J. Appl. Math. Phys. (ZAMP) 30, 315-325.

Saha, P. and Tremaine, S.: 1992, "Symplectic integrators for Solar system dynamics", Astron. J. 104, 1633-1640.

Schubart, J.: 1968, "Long-period effects in the motion of Hilda-type planets", Astron. J. 73, 99-103.

Watson, J.S., Mistretta, G.D., and Bonavito, N.L.: 1975, "An analytical method to account for drag in the Vinti satellite theory", Celest. Mech. 11, 145-178.

Wisdom, J. and Holman, M.: 1991, "Symplectic maps for the N-body problem", Astron. J. $102,1528-1538$. 